\title{
QUESTIONING QUESTIONS IN LANGUAGE, CULTURE AND COGNITION
}

\author{
Simon Borchmann \\ Roskilde Universitet \\ sub@ruc.dk \\ Sune Sønderberg Mortensen \\ Roskilde Universitet \\ sunes@ruc.dk \\ Louise Tranekjær \\ Roskilde Universitet \\ louiset@ruc.dk
}

\section{Why question questions?}

The motivation for questioning questions arose in the research group Language, Culture and Cognition in 2018 when several members were working on material that included questions. In this work, a series of problems appeared, including: How do we classify questions based on their functions? What is the cognitive basis of questions? How do we account for the specific functions that questions serve in activity types? The problems led to consideration as to whether there was a basis for a broader discussion of questions, and when the group invited to the open symposium Questioning Questions in Language, Culture and Cognition, it turned out that there was a widespread interest within the international linguistic research community. At the symposium held at Roskilde University on November 15, 2018, 14 papers were presented, and following the research group's call for papers for a special issue, several new proposals came along - each contributing to the classification, analysis and characterisation of questions. This indicates not only that there is a lively interest in questions, but also that there is a need to discuss and add to the existing classifications, analyses and characterisations of questions. In this issue we have gathered the 11 most relevant contributions.

Questions have been investigated within language sciences from pragmatic, cognitive, functional, formal, semantic, conversation analytic, interaction analytic and sociolinguistic approaches. Question-response systems in a 
variety of languages have been proposed (e.g. Heineman 2010, Stivers 2010). Indeed, a volume about questions has been published in the series Language, Culture and Cognition (de Ruiter 2012) (without relation to the research group at Roskilde University). Yet, the apparent need to discuss and add to the existing research can be seen as a result of a general development of linguistics and, specifically, as a consequence of the specific construal of the juxtaposition language, culture and cognition that provides the framework for this issue.

As to the general development, the pragmatic turn of the 70's involved a change from a focus on the forms of language to the use we make of the forms. At the same time, the empirical basis expands from limiting itself to a small set of constructed, well-formed sentences to include actual everyday language use in various forms. These alterations were accompanied by an awareness of the diversity and change of language, both in terms of form and function and in terms of systems (Drucker 2012). Thereby, the pragmatic turn fostered a series of new linguistic problems. While the first enlargement of the empirical basis was modest and generally confined itself to literary prose and conversation (Linell 2004, Borchmann 2019), linguistic forms within more and more specific genres, interaction types, institutions and activities were added. In parallel with the continuous expansion of the empirical basis, the analysis of language use develops into sophisticated distinctions between types of meaning, including abstract meaning, entailments, presuppositions, conventional implicatures, general implicatures and particular implicatures, and the linguistic object extends to also include genre and interaction typespecific meaning. This expansion and refinement have contributed to the recognition of the diversity of language use and the complexity of formfunction relations. At the same time, it raises a series of new issues. The pragmatic perspective thus creates a continuous need for new classifications, analyses and characterisations.

All articles in this issue can, in general, be regarded as addressing the problems that the pragmatic turn has created and continuously creates. Thus, the articles focus on the functions of questions. The empirical basis for classifications, analyses and characterisations encompasses such diverse genres and types of interaction as psychotherapy, storytelling in aided communication, courtroom interaction, executive coaching, police interviews, political press interviews, whiteboard meetings in workplace settings, news articles, and pilot-to-pilot communication in soaring. The functions characterised include general functions as well as genre and interaction type-specific functions and activity-crossing functions. Overarchingly, the contributions share an interest in the diversity of language use and recognise the complexity of form-function relations.

\section{Language, culture and cognition}

More specifically, the motivation for questioning questions can be seen as a result of the perspective brought about by the juxtaposition language, culture 
and cognition. This perspective, which characterises the research group Language, Culture and Cognition, involves an understanding of cognition that differs from the one on which cognitive linguistics has been based. Cognition is not regarded as an abstract and individual mental process, but as a concrete collective process in which the individual participates (Reed 1996). Epistemically, cognition is "neither copying nor construction"; cognition is "the process that keeps us active, changing creatures in touch with an eventful, changing world" (Reed 1996:13). Hence, cognition is not something that happens to us, but something we do; and it includes not only the use of our brain, but our entire perceptual system, including our eyes, head, body, arms and legs, and the opportunities it offers to move around in the environment and create information (Gibson 1986/1979). As a collective process, cognition is distributed (Hutchins 1995) among people and tools, and embedded in more or less well-defined activities or forms of life. That is, cognition is a culture-based process (Hutchins 1995). The basic linguistic assumption, then, is that language use is embedded in such culture-based processes and must be explained and described with reference to extra-linguistic factors, including values and social norms, culture-specific habits and standards, tools and technologies, knowledge of the world and practical, non-linguistic and pre-linguistic experience and abilities. This relation of embeddedness is both functional and dynamic. The functional aspect implies that the forms and patterns we observe are assumed to be as they are because they serve a more or less general function, in a more or less well-defined system. The dynamic aspect implies that the use of forms is ontologically primary, and that variance even on very short timescales can cause changes both within the system and of the system. This perspective does not, however, change the ambition of identifying regularities in form and function and discovering systems. But it stresses that the above-mentioned extra-linguistic factors must be taken into account and, thereby, suggests an interdisciplinary approach relying on linguistics, psychology, sociology and anthropology.

Applying the outlined perspective to questions raises a series of new problems: If cognition is a distributed, culture-based process, what is the cognitive basis of questions? What are the functions of questions embedded in such processes? What is the relation between the forms and the functions of such questions? How can questions bring about change in and of systems? Not all the contributions in the issue share the theoretical basis of the outlined perspective, nor do they necessarily address the problems mentioned above in explicit ways. Moreover, since the issue does not strive for terminological uniformity, in some articles distributed cognition is described in terms of common ground management or intersubjectivity, and in some articles functions are described in terms of tasks. However, all the articles contribute to the solution of the problems that the perspective language, culture and cognition raises, and as indicated by the cross-references, all the articles take views of one or more of the other articles into consideration. 


\section{Themes and contributions}

The contributions are grouped into three sections under three overarching themes. The first section, Class, form and function, includes three contributions that address issues related to classification and analysis of questions and identification of stable relations between form and general functions.

Nielsen presents a systematic classification of speech acts. Based on four parameters of preparatory conditions, Nielsen expands Searle's rudimentary analysis of questions, and suggests ten distinct types of interrogative speech acts. Thereby, the article gives an overview of the diversity of the subjectmatter, and raises a number of issues to be scrutinised in the subsequent 10 articles.

Borchmann points to a discrepancy between Searle's analysis of speech acts and the semantic structure of three questions in an everyday activity. Tracing the discrepancy back to the cognitive basis of Searle's analysis, Borchmann compares Searle's theory of intentionality and perception to cognitive ethnographic observations of the situations that gave rise to the three questions. On that basis, Borchmann claims that Searle's analysis is based on intentionality and perception in idle, whereas the semantic structure of interrogative speech acts in an everyday activity is a product of intentionality and perception in operation.

Heim and Wiltschko take a starting point in the diversity of form and function that characterises the phenomenon associated with questionhood within linguistics. In continuation of this, they propose a decomposition of the illocutionary force of questions into two variables: commitment and engagement. Commitment covers the speaker's readiness to publicly commit to the truth of a proposition; engagement can be defined as the speaker's expectation of how the addressee will respond to the speaker's utterance. They then proceed to show how these variables are encoded in English syntax and intonation.

The second section, Multifunctionality and context, comprises five contributions, four identifying and describing genre-specific, interaction type-specific or activity-crossing functions of questions, and one focusing on deceptive or evasive answers from a verbal as well as non-verbal analytical perspective.

Tranekjær explores how team leaders in an industrial laundry facility use the multifunctionality of questions as an interactional resource to simultaneously ensure, on the one hand, the participation and understanding of migrant employees and, on the other, the progression and efficiency of the meeting and the production more generally.

The article by Mayes and Clinkenbeard also addresses the way in which questions can be used to manage the opposing agendas of securing intersubjectivity and progression, though within the context of aided communication. They show how questions can be used as a recipient 
resource to support and progress the narrative production of speakers with communication disabilities.

Finkbeiner investigates how the illocutionary force of wh-headlines that is, a specific syntactical form in a specific sequential position - is to be specified, and how it can be accounted for. Finkbeiner suggests an analysis of wh-headlines as both backward- and forward-referring means that fulfil the two main functions of headlines, namely, to arouse the reader's interest and to direct the reader's attention to the subsequent text. The object is whheadlines in German; however, a similar phenomenon may be observed in other languages, including English, Swedish, Danish and French.

Collin's case study of simulated investigative interviews in a Danish police training context combines pragmatic, pragma-dialectical and functional grammatical perspectives. Specifically, the study focuses on the functions of reconstructive speech acts in the interviews, i.e. questions involving repetition, paraphrases or (re)formulations of previous statements made by the mock suspects being interviewed. Collin documents how the reconstructive questions play an important part in the interactional management of the two quite different activity types going on concomitantly, viz., the interview activity and the training activity.

Archer illustrates how a multi-channel approach to the analysis of questioning, incorporating the study of facial expressions, body movements and gestures in addition to traditional linguistic and interactional approaches, can generally augment the analysis and be particularly instrumental for detecting and describing deceptive and evasive language use. Investigating questioning sequences from two different interview contexts, a police interview and a political press briefing, Archer discusses a variety of salient cues in the verbal and non-verbal behaviour of the interviewees as they respond to potentially incriminating questions.

The third section, Systems, variation and change, includes three contributions each suggesting or adopting a global perspective on questioning sequences, and accounting for the relation between the local and the global level of dialogical systems that serve specific purposes.

Graf, Dionne and Spranz-Fogasy make a programmatic argument for an interdisciplinary approach to the transformative potential of questions in executive coaching sessions, proposing a model for the analysis of both local and global change patterns, based on the combination of conversation analysis and psychological coaching theory.

Mortensen approaches and challenges the well-described issue of discursive control and coercion in questioning through comparing the uses of questions by prosecutors and lawyers in American and Danish trial examinations. Applying structural-functional as well as pragmatic classification criteria, close to 800 trial questions are studied and analysed from both quantitative and 
qualitative perspectives. Mortensen discusses how the observed differences reflect legal cultural differences between USA and Denmark, and differences in how question control and coercion can be understood in the two linguistic and languacultural contexts.

Finally, Worsøe and Jensen's article explores the change potential of questions in psychotherapy sessions from an interactional and ecological perspective, providing insight into the way in which the atypical, yet recurrent, phenomenon of clients posing questions to the therapist influences the dialogical and asymmetric relation between the participants.

\section{References}

Borchmann, Simon. 2019. The spectator bias in the linguistic descriptions of information structure. Language Sciences, vol. 76.

Drucker, Andreas H. 2008. Pragmatics in the history of linguistic thought. Keith Allan \& Kasia M. Jaszczolt (eds.) The Cambridge Handbook of Pragmatics. Cambridge: Cambridge University Press, 495-512.

Gibson, James J. 1986/1979. The ecological approach to visual perception. New York: Psychology Press.

Heinemann, Trine. 2010. The question-response system of Danish. Journal of Pragmatics 42, 2703-2725.

Hutchins, Edwin. 1995. Cognition in the Wild. Cambridge, Massachusetts: The MIT Press.

Linell, Per. 2004. The Written Language Bias in Linguistics: Its Nature, Origins and Transformations. London: Routledge.

Reed, Edward S. 1996. Encountering the world. New York, NY: Oxford University Press.

de Ruiter, Jan P. (ed.). 2012. Questions: Formal, Functional and Interactional Perspectives. (Language, Culture and Cognition). Cambridge: Cambridge University Press.

Stivers, Tanya. 2010. An overview of the question-response system in American English conversation. Journal of Pragmatics 42, 2772-2781. 